Cite this: Phys. Chem. Chem. Phys., 2012, 14, 5489-5503

\title{
Vibrations and hydrogen bonding in porphycene $\dagger$
}

\author{
Sylwester Gawinkowski, ${ }^{a}$ Lukasz Walewski, ${ }^{b}$ Alexander Vdovin, ${ }^{c}$ \\ Alkwin Slenczka, ${ }^{c}$ Stephane Rols, ${ }^{d}$ Mark R. Johnson, ${ }^{d}$ Bogdan Lesyng ${ }^{e f}$ and \\ Jacek Waluk $* a$
}

Received 22nd December 2011, Accepted 20th February 2012

DOI: $10.1039 / \mathrm{c} 2 \mathrm{cp} 24098 \mathrm{j}$

Combined use of IR, Raman, neutron scattering and fluorescence measurements for porphycene isolated in helium nanodroplets, supersonic jet and cryogenic matrices, as well as for solid and liquid solutions, resulted in the assignments of almost all of 108 fundamental vibrations. The puzzling feature of porphycene is the apparent lack of the $\mathrm{N}-\mathrm{H}$ stretching band in the IR spectrum, predicted to be the strongest of all bands by standard harmonic calculations. Theoretical modeling of the IR spectra, based on ab initio molecular dynamics simulations, reveals that the $\mathrm{N}-\mathrm{H}$ stretching mode should appear as an extremely broad band in the $2250-3000 \mathrm{~cm}^{-1}$ region. Coupling of the $\mathrm{N}-\mathrm{H}$ stretching vibration to other modes is discussed in the context of multidimensional character of intramolecular double hydrogen transfer in porphycene. The analysis can be generalized to other strongly hydrogen-bonded systems.

\section{Introduction}

Specific spectral and photophysical characteristics, as well as an essential role played by porphyrins in many biological processes, make these compounds attractive for promising applications in various fields. ${ }^{1}$ Porphycene $(\mathrm{Pc})$, a constitutional isomer of porphyrin (Scheme 1), differs from the latter in several aspects. The electronic absorption of Pc and its derivatives in the low energy range is about an order of magnitude stronger than in the parent compound; a similar enhancement is observed for fluorescence quantum yield. ${ }^{2,3}$ On the other hand, triplet state formation is less efficient, but sufficient enough to generate singlet oxygen with yields exceeding $30 \%$, which makes porphycenes good candidates for photodynamic therapy. ${ }^{4}$

With regard to fundamental research, tautomerism in porphyrins and porphycenes has attracted much attention. ${ }^{5-36}$

${ }^{a}$ Institute of Physical Chemistry, Polish Academy of Sciences, Kasprzaka 44,01-224 Warsaw, Poland.E-mail: waluk@ichf.edu.pl; Fax: + 4822 3433333; Tel: + 48223433332

${ }^{b}$ Interdisciplinary Centre for Mathematical and Computational Modelling, University of Warsaw, Pawińskiego 5a, 02-106 Warsaw, Poland.E-mail: ljw@icm.edu.pl

${ }^{c}$ Institut für Physikalische und Theoretische Chemie, Universität Regensburg, Universitätsstraße 31, 93053 Regensburg, Germany. E-mail: Alkwin.Slenczka@chemie.uni-regensburg.de

dInstitut Laue-Langevin, Grenoble,France.E-mail: johnson@ill.fr

${ }^{e}$ Department of Biophysics, Faculty of Physics, University of Warsaw, Zwirki i Wigury 93, 02-089 Warsaw, Poland.

E-mail: B.Lesyng@icm.edu.pl

${ }^{f}$ Bioinformatics Laboratory, Mossakowski Medical Research Centre, Pawinskiego 5, 02-106 Warsaw, Poland

$\dagger$ Electronic supplementary information (ESI) available: Details of normal mode calculations, computed vibrational frequencies, forms of vibrational modes. See DOI: 10.1039/c2cp24098j

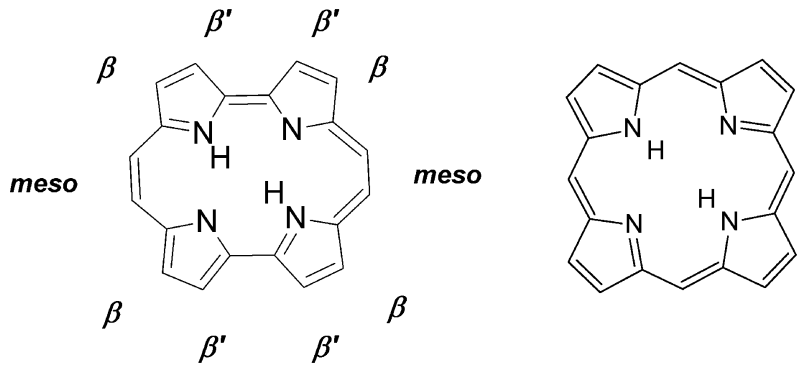

Scheme 1 Porphycene (left) and porphyrin (right).

Both shape and size of the internal cavity formed by four nitrogen atoms are different in the two molecules, which results in the two intramolecular $\mathrm{N}-\mathrm{H} \cdots \mathrm{N}$ hydrogen bonds being much stronger for Pc. This has immense consequences for the tautomerization rates. The two internal hydrogens in Pc move back and forth between two chemically equivalent trans structures (Scheme 2) with the rate of $5.8 \times 10^{11} \mathrm{~s}^{-1}$ at room temperature. ${ }^{22}$ The corresponding rate for porphyrin is many orders of magnitude lower $\left(2600 \mathrm{~s}^{-1}\right.$ at $\left.267 \mathrm{~K}\right){ }^{8}$ Also the mechanisms of tautomerization are different. In porphyrin, the reaction proceeds in a stepwise manner, starting from thermally activated tunneling of single hydrogen. This leads to the cis tautomer, which, upon second single hydrogen transfer can form the final trans tautomer, or revert to the initial trans form. ${ }^{5-7}$ The experimental and theoretical evidence for porphycene suggests a concerted, albeit not necessarily synchronous mechanism. ${ }^{16-18,22,29,30}$ Actually, for mesosubstituted porphycenes, where both trans and cis-1 forms were detected, the experiment showed that the trans-trans conversion is faster than the cis-trans process. ${ }^{19}$ 

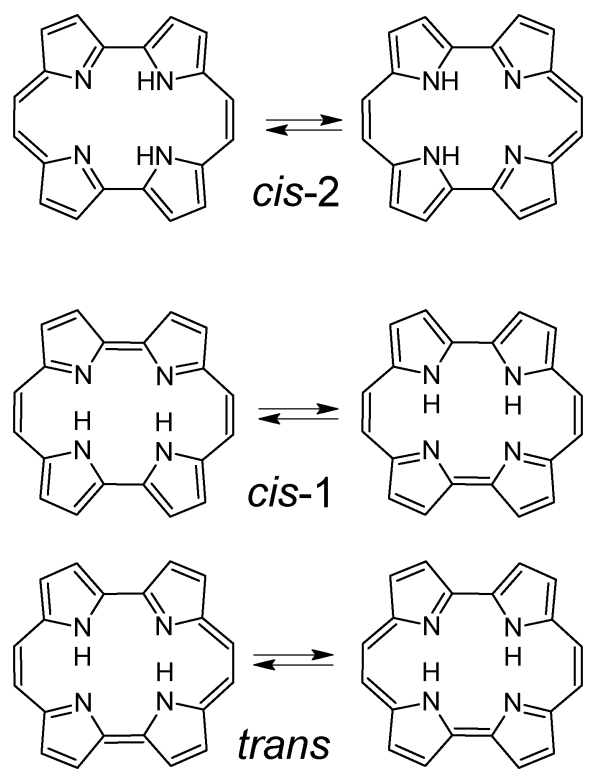

Scheme 2 Tautomeric forms of Pc.

The studies of $\mathrm{Pc}$ in the regime of molecular isolation, either in supersonic jets ${ }^{15,31}$ or in helium nanodroplets, ${ }^{20}$ revealed tunneling splittings in electronic absorption and fluorescence, due to delocalization of the internal hydrogens. These splittings are vibrationally mode-selective. For some vibrations the value of splitting, $4.4 \mathrm{~cm}^{-1}$, is practically the same as that determined for the $0-0$ transition. However, for a low frequency $180 \mathrm{~cm}^{-1}$ mode, the splitting exceeds $12 \mathrm{~cm}^{-1}$, the increase reflecting the form of the vibration, which brings the hydrogen-bonded $\mathrm{N}$ atoms closer to each other, thus lowering the tautomerization barrier (see ESI $\dagger$ or Fig. 8 for atomic displacements from the equilibrium structure in this mode, $2 \mathrm{AG}$ ). For yet another low frequency mode, which effectively decreases the hydrogen bonding strength (1 AG, ESI $\dagger$ or Fig. 8), the splitting becomes undetectably low $\left(<0.1 \mathrm{~cm}^{-1}\right)$. These findings point to the important role of tunneling and indicate a multidimensional character of tautomerization in porphycene, confirmed by theoretical studies. ${ }^{18,24,27,34-36}$ In a work devoted to identifying vibrational modes relevant for the proper description of the potential governing the motion of the inner protons, Kühn and co-workers used four-dimensional and six-dimensional nuclear wavefunctions, the latter for a singly deuterated isotopologue. ${ }^{35}$

Naturally, the key to understanding the complex mechanism of tautomerization in $\mathrm{Pc}$ is the knowledge of the intramolecular hydrogen bond (HB) parameters. Usually, a measure of the HB strength is provided by the frequency of the $\mathrm{N}-\mathrm{H}$ stretching vibration. Surprisingly, previous IR studies of porphycene $^{10}$ revealed no trace of the $\mathrm{N}-\mathrm{H}$ stretching band, even though it was predicted by both DFT and RHF calculations to be the most intense in the whole IR spectrum. Moreover, the authors stated that the IR, Raman and fluorescence data are not conclusive enough to determine whether the trans form is really dominant, as suggested by the calculations.

The experiments based on polarization spectroscopy performed for Pc in the last decade ${ }^{12,14,22,25,29,30,32,33}$ leave little doubt that the lowest energy form corresponds to the trans tautomer, at least for parent Pc. However, except for a series of papers devoted to the resonance Raman spectra of porphycene anions, ${ }^{37-40}$ no progress has been reported regarding vibrational structure. The goal of the present work was, therefore, to assign in detail the vibrations in porphycene, with particular attention to the modes involved in the intramolecular hydrogen bonds. For this purpose, we have performed IR, Raman, fluorescence and inelastic neutron scattering (INS) experiments for the parent Pc and for its doubly-deuterated isotopologue, Pc- $d_{2}$, in which the inner protons were replaced by deuterons. Regarding theoretical studies, going beyond the harmonic model seemed mandatory. We therefore carried out extensive ab initio molecular dynamics simulations, aimed at understanding coupling between vibrational modes in porphycene.

The results allow us to propose a solution for the porphycene puzzle, to understand the apparent lack of a strong $\mathrm{N}-\mathrm{H}$ band in the IR spectrum, and to discuss the coupling between low and high frequency modes in porphycene.

\section{Experimental and theoretical methods}

Porphycene was synthesized and purified according to literature procedures. ${ }^{41,42}$ The doubly deuterated isotopologue was obtained by dissolving the compound in the $\mathrm{CH}_{3} \mathrm{OD} / \mathrm{CHCl}_{3}$ mixture and subsequent evaporation of the solvent. Perdeuteration of $\mathrm{Pc}$ was achieved by heating the compound in $70 \% \mathrm{D}_{2} \mathrm{SO}_{4}$ in $\mathrm{D}_{2} \mathrm{O}$ for $24 \mathrm{~h}$. After that, chloroform was added to the cooled solution before being neutralized with diluted $\mathrm{NaOD} / \mathrm{D}_{2} \mathrm{O}$. Finally, the organic layer was concentrated in vacuo. The degree of deuteration was checked by mass spectra and was estimated to be higher than $90 \%$.

The solvents $\left(\mathrm{CCl}_{4}\right.$ and $\mathrm{CS}_{2}$, IR grade, acetone, spectroscopic grade, all from Aldrich) were used without further purification. $\mathrm{KBr}$ powder (IR grade, Aldrich) was dried for $4 \mathrm{~h}$ at $250{ }^{\circ} \mathrm{C}$ before use.

The infrared spectra were measured on a Nicolet Magna 560 FT spectrometer, equipped with a MCT/B liquid-nitrogencooled detector or on a Nicolet 170SX, using InSb 2 and MCT liquid-nitrogen-cooled detectors. For the far-IR region, the measurements were made using a Nicolet $20 \mathrm{~F}$ FT spectrometer with a DTGS (room temperature) detector using polyethylene pellets.

At room temperature, the spectra were recorded for solutions, $\mathrm{KBr}$ or polyethylene pellets, or thin solid films on $\mathrm{KBr}$ windows. The spectra at low-temperature were obtained for Pc embedded in solid argon, nitrogen or xenon, obtained by deposition of matrices on a cold $\mathrm{KBr}$ window of a closed-cycle helium cryostat (CSW-202 N, Advanced Research Systems). A typical guest-host ratio was about $1: 10^{3}$. The deposition could be monitored by on-line collection of IR spectra.

The Raman spectra were taken with an InVia Renishaw Raman spectrometer based on a Leica microscope equipped with $100 \times, 50 \times, 20 \times, 5 \times$ objectives for visible and a $15 \times$ objective for UV excitation, 1200, 1800 and 2400 grooves $\mathrm{mm}^{-1}$ gratings, and a thermoelectrically cooled CCD array detector. Three laser lines were used: $785 \mathrm{~nm}$ (HPNIR785), $514.5 \mathrm{~nm}$ (Stellar Pro argon Modu-Laser, LLC), and $325 \mathrm{~nm}$ (He-Cd Kimmon laser). The laser power on the sample was kept below $0.1 \mathrm{~mW}$. The spectral resolution was $5 \mathrm{~cm}^{-1}$ and the wavenumber accuracy was $\pm 2 \mathrm{~cm}^{-1}$, both calibrated with the Rayleigh line 
and the $520.6 \mathrm{~cm}^{-1}$ line of silicon. The samples were checked for thermal decomposition; whenever necessary, it was avoided by defocussing or lowering the laser power.

Dispersed emission spectra of Pc- $d_{0}$ and Pc- $d_{2}$ were taken in a helium droplet machine. The droplet source was built according to the design developed in Göttingen. ${ }^{43,44}$ Helium droplets were generated in a supersonic expansion of helium gas (grade 6.0) through a $5 \mathrm{~mm}$ orifice. The stagnation pressure was 20 bar and the source temperature was $11 \mathrm{~K}$, which leads to an average droplet size of 20000 helium atoms. ${ }^{45}$ Pc was doped into the helium droplets by the pick-up technique. Thereby, the droplet beam was passed through an oven, where a solid sample of Pc was heated for sublimation. The temperature was tuned to optimize for single molecule doping of the droplets. Further downstream, the doped droplet beam was irradiated by a single mode ring dye laser (Coherent 899-29 Autoscan II) tuned to the electronic origin of the dopant species $\left(16145.92 \mathrm{~cm}^{-1}\right.$ for Pc- $d_{0}$, and $16101.67 \mathrm{~cm}^{-1}$ for Pc- $d_{2}$ ). The fluorescence was collected by a quartz lens and imaged onto the entrance slit of a grating spectrograph (SPEX). The detector was a charge coupled device (CCD) camera (Andor, DU 401-BV) with a $256 \times 1024$ CCD chip operated in the vertical binning mode. Thus, a spectrum consisting of 1024 data points was accumulated with a spectral resolution of $0.7 \mathrm{~cm}^{-1}$ per pixel. The frequency scale was calibrated with an Ar-Ne lamp using the literature data. ${ }^{46}$

INS spectra were measured on two spectrometers. The direct geometry, thermal neutron, time-of-flight (TOF) spectrometer, IN $4,{ }^{47}$ was used to measure the low frequency spectrum, including the elastic peak. A Cu220 monochromator was used to give an incident wavelength of $0.9 \AA(E=100 \mathrm{meV})$ allowing Stokes' spectrum to be measured up to almost $100 \mathrm{meV}$ in the temperature range from $10 \mathrm{~K}$ to $300 \mathrm{~K}$. In this configuration the energy resolution (FWHM) is about $3 \%$ of the energy transfer. The neutron TOF is used to measure the energy of scattered neutrons. The pyrolitic graphite monochromator (PG002) was used to give an incident wavelength of $2.7 \AA(E=11 \mathrm{meV})$ and a better energy resolution (FWHM) of $\sim 1 \mathrm{meV}$ which is approximately constant across the spectrum. With lower energy neutrons, anti-Stokes' spectrum was measured in the temperature range from $150 \mathrm{~K}$ to $300 \mathrm{~K}$ for which the excited vibrational levels are sufficiently wellpopulated up to about $50 \mathrm{meV}$.

Spectra from $30 \mathrm{meV}$ up to $500 \mathrm{meV}$ were measured using the inverse geometry, beryllium filter spectrometer, IN1. ${ }^{48}$ The final energy is fixed by the beryllium filter at $3 \mathrm{meV}$. A spectrum is obtained by varying the incident wavelength, which is achieved by scanning the take-off angle of the crystal monochromator, in this case $\mathrm{Cu} 220$. Energy resolution (FWHM) is approximately $2 \%$ of energy transfer. Spectra were measured at $10 \mathrm{~K}$ and $200 \mathrm{~K}$.

Samples were mounted in aluminium sachets of height and width (4 $\mathrm{cm}$ and $3 \mathrm{~cm}$, respectively) chosen to intercept the whole neutron beam. Sample thickness was not more than $1 \mathrm{~mm}$. Reflection geometry for the sample was chosen, its orientation being $135^{\circ}$ to the incident beam. Standard orange cryostats were used for temperature control, the precision being better than $\pm 1 \mathrm{~K}$ at all temperatures. The four isotopologues, Pc- $d_{0}$, Pc- $d_{2}$, Pc- $d_{12}$ and Pc- $d_{14}$, were measured on both spectrometers. The data treatment was performed using the LAMP program, ${ }^{49}$ in particular for the integration of IN4 data to produce generalized densities of states.

Quantum-chemical calculations were carried out using Gaussian 03 and Gaussian 09 program packages. The equilibrium geometry of $\mathrm{Pc}$ in the electronic ground state was optimized at the B3LYP/6-31G(d,p) and B3LYP/6-311++G(fd,2dp) levels. This was followed by harmonic frequency calculations and normal mode analysis (NMA). Simulated fluorescence spectra were obtained using the Franck-Condon approximation as implemented in Gaussian 09 package. We will refer to the results obtained with those methods as "harmonic", opposed to "anharmonic", as discussed later in the text.

Born-Oppenheimer molecular dynamics simulations were carried out at the DFT level of theory as implemented in the CPMD package. ${ }^{50}$ Gradient-corrected exchange-correlation functional (BLYP) was used. ${ }^{51,52}$ The valence electron wavefunction was expanded in the plane wave (PW) basis up to $70 \mathrm{Ry}$, and the interactions of core electrons were described using the norm conserving pseudopotentials. ${ }^{53}$ The Poisson equation was solved using the Hockney method ${ }^{54}$ in a simple cubic simulation cell with the side length of $15 \AA$ A. Decoupling of the periodic images for the electrostatic interactions was applied using cluster boundary conditions. ${ }^{69}$ The 3 rd order predictorcorrector extrapolation of the wave function was applied to decrease the number of SCF cycles required to converge wavefunction gradients down below $5 \times 10^{-6}$ a.u. Nuclear equations of motion were integrated using the symplectic velocity Verlet algorithm with the time-step of 20 a.u. ( $\sim 0.48 \mathrm{fs}$ ). Center of mass motion was subtracted every $0.5 \mathrm{ps}$.

The IR spectra were computed according to the following protocol. The system was initially equilibrated at $300 \mathrm{~K}$ using one Nosé-Hoover chain thermostat of length 3 per degree of freedom. ${ }^{55} 100$ ps long NVT trajectory was generated using the same massive thermostating scheme. The positions and velocities were selected from this trajectory at 2 ps intervals and used as initial conditions for 45 independent NVE simulations, each 20 ps long. The total dipole moment was computed at each MD step along all the NVE runs. In the post-processing phase the Fourier transform of the classical autocorrelation function of the total dipole moment was computed for each trajectory. The quantum correction factor was applied to obtain the IR lineshape function. ${ }^{56}$ The resulting IR spectra were averaged over all 45 NVE simulations providing the canonical ensemble average at $300 \mathrm{~K}$. The same protocol was applied to three Pc isotopologues, Pc- $d_{0}$, Pc- $d_{1}$ and Pc- $d_{2}$. Normal mode analysis (NMA) was carried out using the same settings as those used for MD simulations, yielding normal mode frequencies $f_{i}$ (see Table $\mathrm{S} 1$, ESI $\dagger$ ) and normal mode vectors $\boldsymbol{e}_{i}$. The vectors $\boldsymbol{e}_{i}$ were assigned to those obtained at the B3LYP/6-31G(d,p) level to obtain a consistent mapping between the theoretical and the experimental vibrations (see $\mathrm{ESI} \dagger$ for the details of this assignment).

Mass-weighted projections $p_{i}(t)$ of the molecular trajectories $\boldsymbol{r}(t)$ onto the normal mode vectors $\boldsymbol{e}_{i}$ were computed according to the formula $p_{i}(t)=\boldsymbol{M r}(t) \cdot \boldsymbol{e}_{i}(\boldsymbol{M}$ being the diagonal matrix of square roots of atomic masses $\left.\sqrt{ } m_{i}\right)$ to obtain 108 projected normal mode (PNM) signals. Power spectra $p_{i}(\omega)$ of the PNMs were computed using Fourier transform, and the dominant 
peaks were fitted with Lorentz functions. Median $\mu_{i}$ of the $i$ th fit was taken as the effective frequency $\nu_{i}=\mu_{i} / 2 \pi$ corresponding to the mode $i$ (see Table S1, ESI $\dagger$ ). The frequencies $\nu_{i}$ match the IR peak positions and can be used to assign molecular motions $\boldsymbol{e}_{i}$ to the IR spectral features. We name these frequencies anharmonic because they were obtained without invoking harmonic approximation to the electronic potential.

\section{Results and discussion}

Porphycene has 108 vibrations (see ESI $\dagger$ for the forms of all normal modes). The $C_{2 \mathrm{~h}}$ symmetry of the planar trans tautomeric forms dictates that eighteen $\mathrm{AU}$ and thirty six $\mathrm{BU}$ modes should be IR-active, whereas in the Raman and fluorescence spectra, transitions involving thirty seven AG and seventeen BG modes are allowed. Inspection of IR and Raman spectra shows that the mutual exclusion principle is strictly obeyed, which provides a strong argument for the trans structure of Pc. This finding also allows a separate analysis of modes of gerade and ungerade symmetry.

\subsection{IR-active vibrations}

Comparison of the experimental results and the theoretical predictions is presented in Table 1 and Fig. 1 and 2. The IR spectra have been previously reported for $\mathrm{Pc}$ embedded in $\mathrm{KBr}$ and CsI pellets and cryogenic nitrogen matrices. ${ }^{10} \mathrm{We}$ have measured the IR spectra for polycrystalline $\mathrm{Pc}, \mathrm{KBr}$ pellets, low-temperature nitrogen and xenon matrices, and, additionally, the IR absorption for Pc solutions. The latter helps in cases where the observed multiplet structure may be due to matrix sites or crystal splittings. Most importantly, we recorded the spectra of both undeuterated Pc $\left(\mathrm{Pc}-d_{0}\right)$ and the isotopologue in which the inner protons have been replaced by deuterons $\left(\operatorname{Pc}-d_{2}\right)$. Finally, we also carried out initial studies on Pc- $d_{12}$, a molecule with all protons except the two internal ones replaced by deuterons. Besides providing information on vibrations involving $\mathrm{N}-\mathrm{H} / \mathrm{N}-\mathrm{D}$ bonds, comparison of experimental and theoretical results for isotopologues improves the reliability of the assignments, because the isotopic shifts are usually computed with better accuracy than the absolute energies of vibrational transitions. Additional support was provided by the INS spectra, which turned out to be particularly useful in the low energy region. The agreement between observed and calculated transitions is impressive (see Fig. 1 and 2), regarding not only the band locations, but also relative intensities. In the case of harmonic frequencies practically the same scaling factors were obtained for the parent and doubly deuterated $\mathrm{Pc}$, using all the fundamental transitions lying below $1600 \mathrm{~cm}^{-1}$. No significant changes in the predicted spectral patterns were observed when the basis set was increased from $6-31 \mathrm{G}(\mathrm{d}, \mathrm{p})$ to 6-311++G(fd,2dp); slightly larger scaling factors were obtained for the larger basis set, 0.975 for Pc- $d_{0}$ and 0.973 for Pc- $d_{2}$, compared with 0.968 and 0.966 obtained while using 6-31G(d,p). The RMS errors were about $8 \mathrm{~cm}^{-1}$ for Pc- $d_{0}$ and $10 \mathrm{~cm}^{-1}$ for Pc- $d_{2}$. In principle, these errors could have been made even smaller by applying different scaling factors for modes of different type, an approach used before for porphyrin. ${ }^{57-59}$ We did not attempt such a procedure, since it would not lead to changes in the vibrational assignments.
We propose the assignments for 52 out of 54 computed vibrational transitions of ungerade symmetry. One of the missing vibrations, $1 \mathrm{AU}$, is of very low frequency and therefore lies outside the spectral range of our instruments. On the contrary, the other one, $30 \mathrm{BU}$, corresponds to the asymmetric $\mathrm{N}-\mathrm{H}$ stretch and was computed as the most intense IR band. This striking discrepancy between experiment and harmonic calculations prompted us to extend the theoretical studies to the model that goes beyond harmonic approximation. As will be shown below, the apparent lack of the $\mathrm{N}-\mathrm{H}$ stretch in the experimental spectrum can be explained by an extreme breadth of this transition, due to the coupling to other vibrational modes.

We could, on the other hand, identify another vibration involved in the intramolecular hydrogen bond, the asymmetric $\mathrm{N}-\mathrm{H}$ out-of-plane bending (18 AU). It was observed at 964-971 $\mathrm{cm}^{-1}$ (depending on the environment) in Pc- $d_{0}$ and red-shifted by $282 \mathrm{~cm}^{-1}$ in Pc- $d_{2}$, in very good agreement with the computational predictions. The calculations also correctly reproduce the decrease in intensity observed for the deuterated derivative. Interestingly, the band position and intensity of this vibration change markedly in different environments. Such behavior, characteristic of the out-of-plane $\mathrm{N}-\mathrm{H}$ mode, has been described previously. ${ }^{60-62}$

The transition energy for the out-of-plane $\mathrm{N}-\mathrm{H}$ bending mode is exceptionally high, indicating strong $\mathrm{N}-\mathrm{H} \cdots \mathrm{N}$ hydrogen bonds. For dibenzo[b,i][1,4,8,11]tetraaza[14]annulene (TAA), another molecule with the same structural motif of the inner cavity composed of four nitrogens (see Scheme 3), we have observed the corresponding band at $778 \mathrm{~cm}^{-1}$, shifting to $599 \mathrm{~cm}^{-1}$ in the deuterated species. ${ }^{63}$ For porphyrin, B3LYP/ 6-31G $(\mathrm{d}, \mathrm{p})$ calculations predict two vibrations with contributions from the asymmetric $\mathrm{N}-\mathrm{H}$ out-of-plane wagging, located at 748 and $808 \mathrm{~cm}^{-1}$. The experiment shows two modes at 785 and $731 \mathrm{~cm}^{-1}$ which are affected by deuteration. ${ }^{57,64,65}$ In the doubly deuterated porphyrin molecule, a band appears at $540 \mathrm{~cm}^{-1}$ which can be assigned to the $\mathrm{N}-\mathrm{H}$ wagging vibration. In a classic work on the IR spectra of porphyrins ${ }^{66}$ Mason identified the "out-of-plane deformation" bands in the parent and $-d_{2}$ porphyrins at 719 and $537 \mathrm{~cm}^{-1}$, respectively. It is interesting to note very similar values of the shifts in TAA and in porphyrin, but a significantly larger value for porphycene, which provides another argument for high intramolecular HB strength in this molecule.

It is well known for porphyrin that no single transition can be assigned to the $\mathrm{C}-\mathrm{N}-\mathrm{H}$ in-plane bending vibration. Several in-plane modes are affected by deuteration, as evidenced by spectral shifts. ${ }^{64} \mathrm{~A}$ similar behavior is observed for Pc. The IR absorption patterns for Pc- $d_{0}$ and Pc- $d_{2}$ are quite similar in the region below $1100 \mathrm{~cm}^{-1}$, but strongly differ in the $1100-1600 \mathrm{~cm}^{-1}$ range. The calculations predict significant contributions of $\mathrm{N}-\mathrm{H}$ bending for as many as six modes in the range of $1200-1600 \mathrm{~cm}^{-1}$ (Table 1). In Pc- $d_{2}$, the N-D bending mainly contributes to two modes, $11 \mathrm{BU}$ and $12 \mathrm{BU}$, with transition energies around $1000 \mathrm{~cm}^{-1}$. This different distribution of the NH/ND bending contributions is the main reason why the forms of some vibrations in the two isotopologues do not follow a $1: 1$ correlation pattern. According to theoretical studies, most of the modes do not significantly change their form after double deuteration. Those that do, correspond to the in-plane 


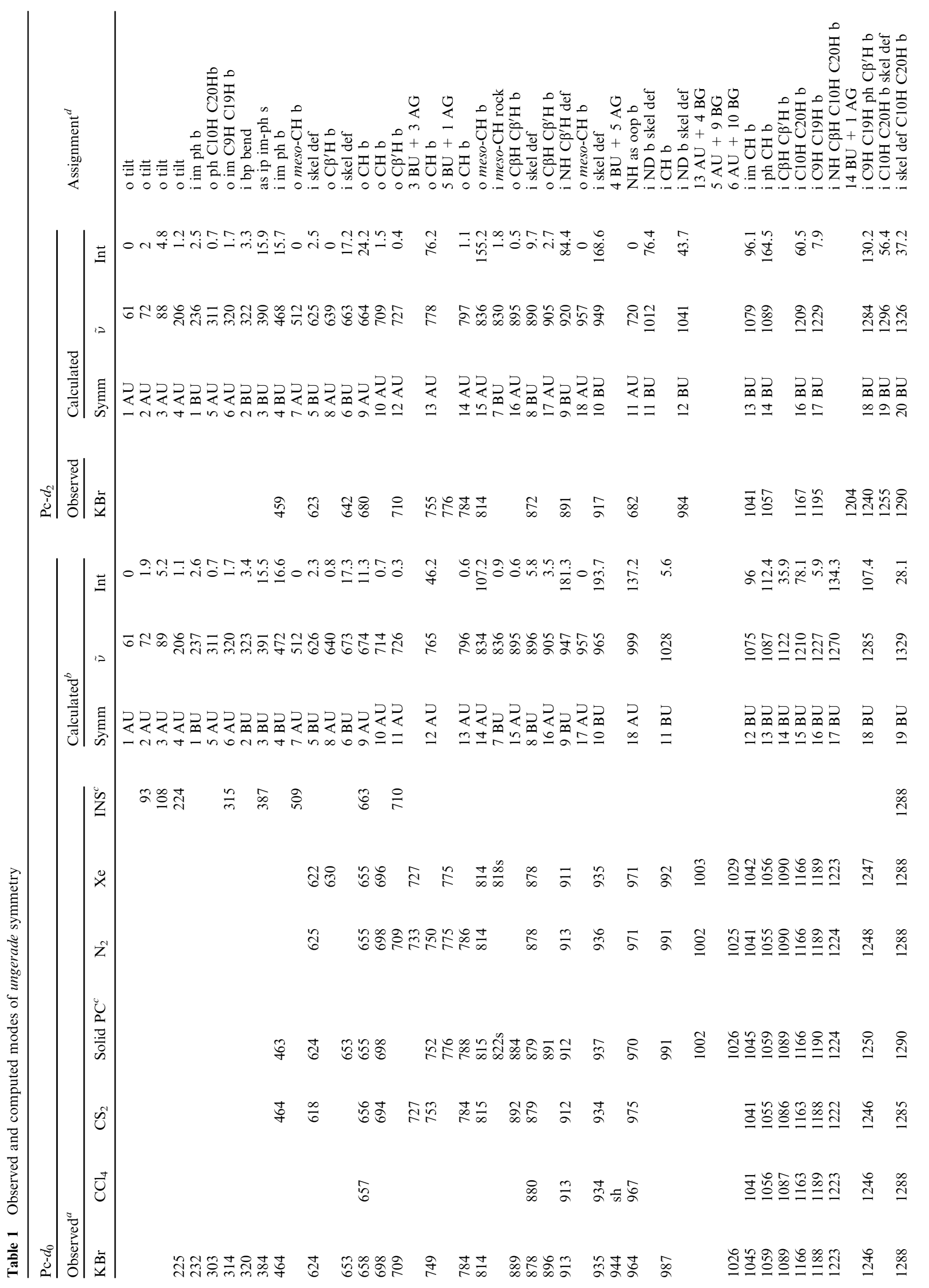




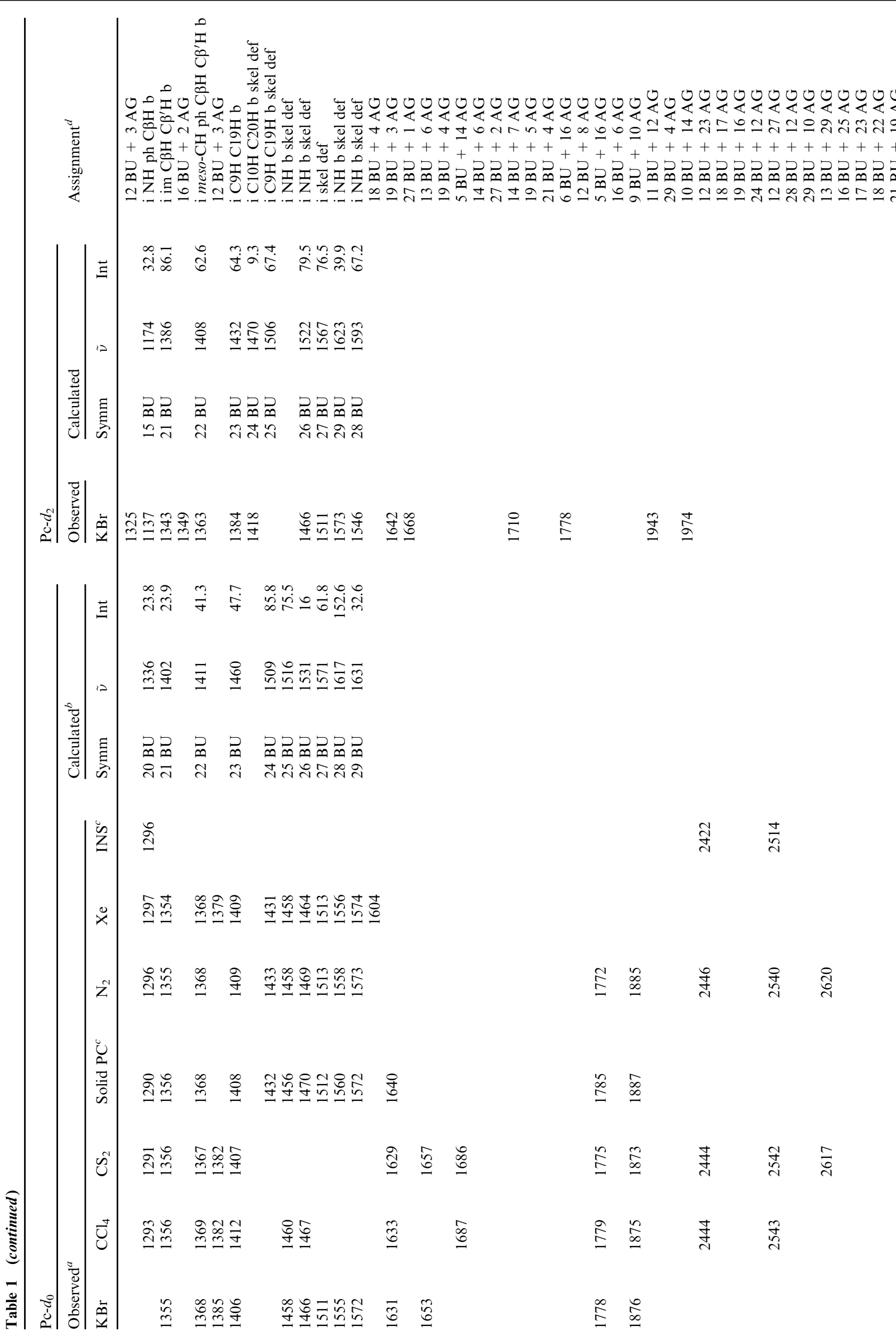




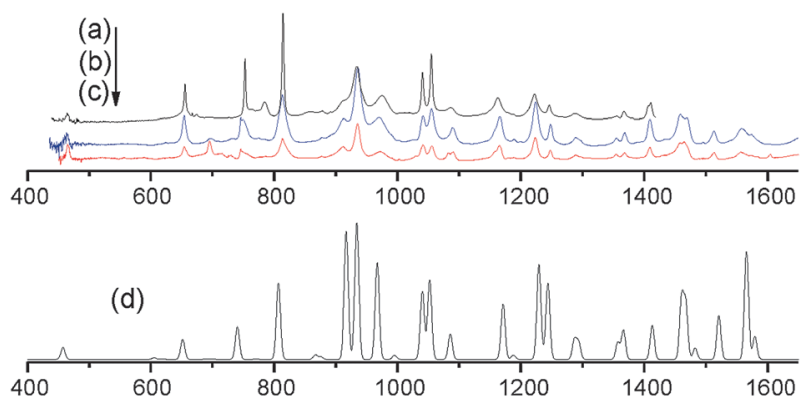

(e)

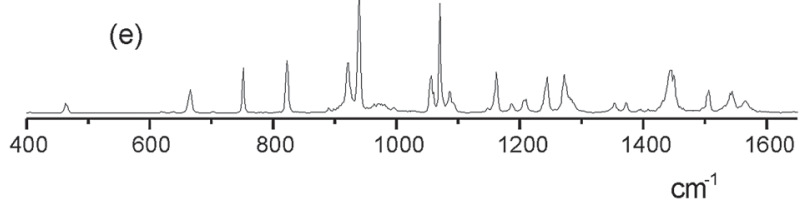

Fig. 1 IR spectra of Pc- $d_{0}$ : experimentally recorded absorption for $\mathrm{CS}_{2}$ solution at $293 \mathrm{~K}$ (a), $\mathrm{N}_{2}$ matrix at $10 \mathrm{~K}$ (b), and Xe matrix at $9 \mathrm{~K}$ (c); harmonic DFT calculations using B3LYP/6-31G(d,p) assuming Gaussian peaks of $4 \mathrm{~cm}^{-1}$ halfwidths for all transitions and a scaling factor of 0.968 (d); ab initio molecular dynamics simulations at $300 \mathrm{~K}$ using BLYP/PW with the scaling factor of 1.032 (e).

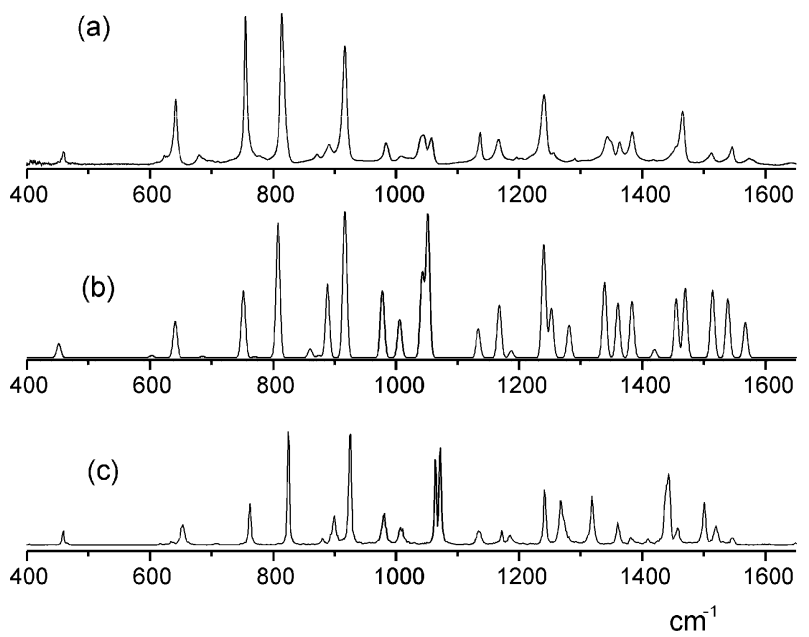

Fig. 2 IR spectra of Pc- $d_{2}$ : experimentally measured absorption in a $\mathrm{KBr}$ pellet at $293 \mathrm{~K}$ (a); harmonic DFT calculations using B3LYP/6-31G(d,p) assuming Gaussian peaks of $4 \mathrm{~cm}^{-1}$ halfwidths for all transitions with the scaling factor of 0.966 (b); ab initio molecular dynamics simulations at $300 \mathrm{~K}$ using BLYP/PW with the scaling factor of 1.032 (c).<smiles>C1=CNc2ccccc2N/C=C\C=Nc2ccccc2N1</smiles>

Scheme 3 Dibenzo[b,i][1,4,8,11]tetraaza[14]annulene (TAA).

BU vibrations. In Tables 1 and 2 we placed in separate rows modes coming from the isotopologues for which no obvious $1: 1$ correlation could be found between their vibrational forms. 


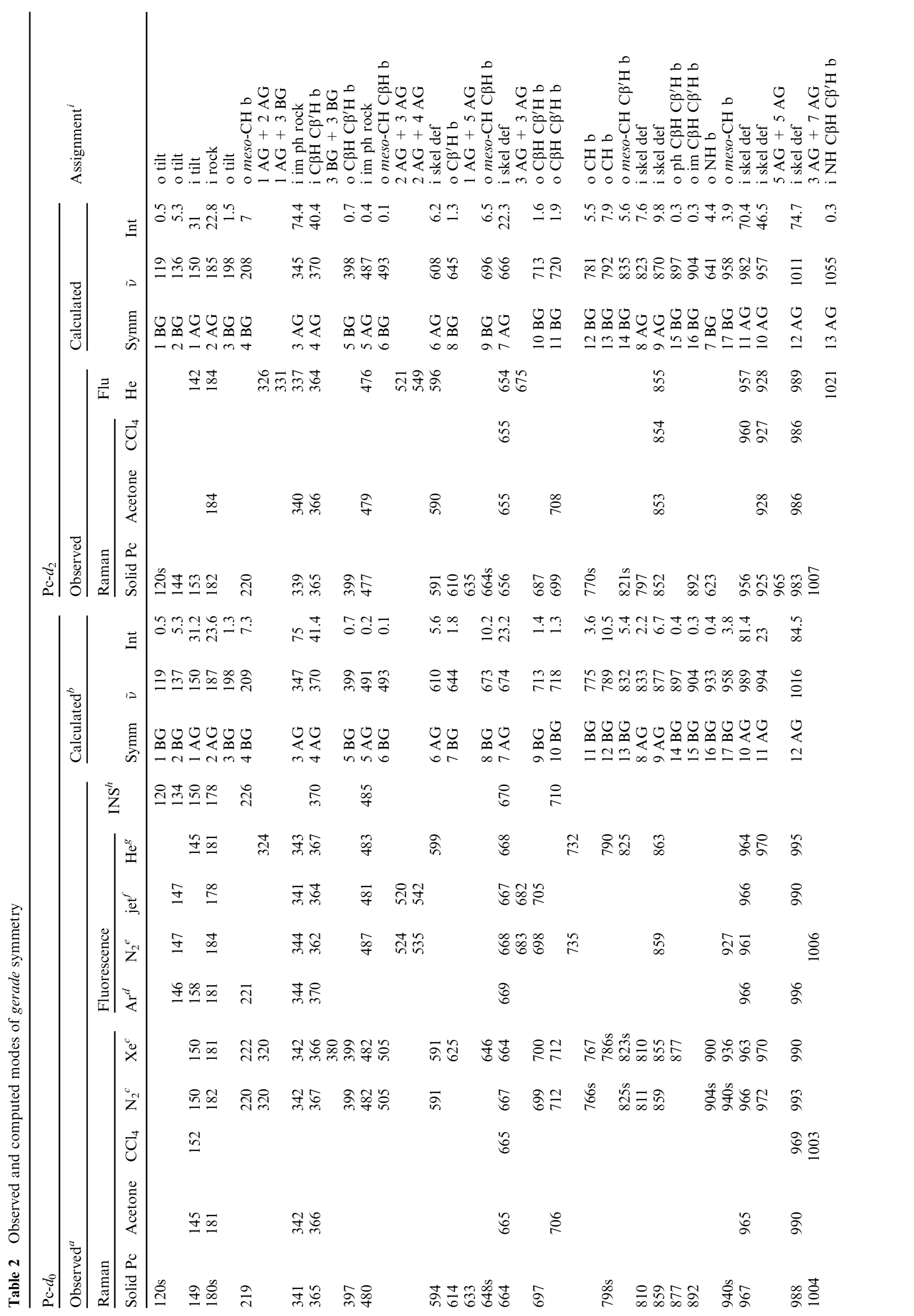




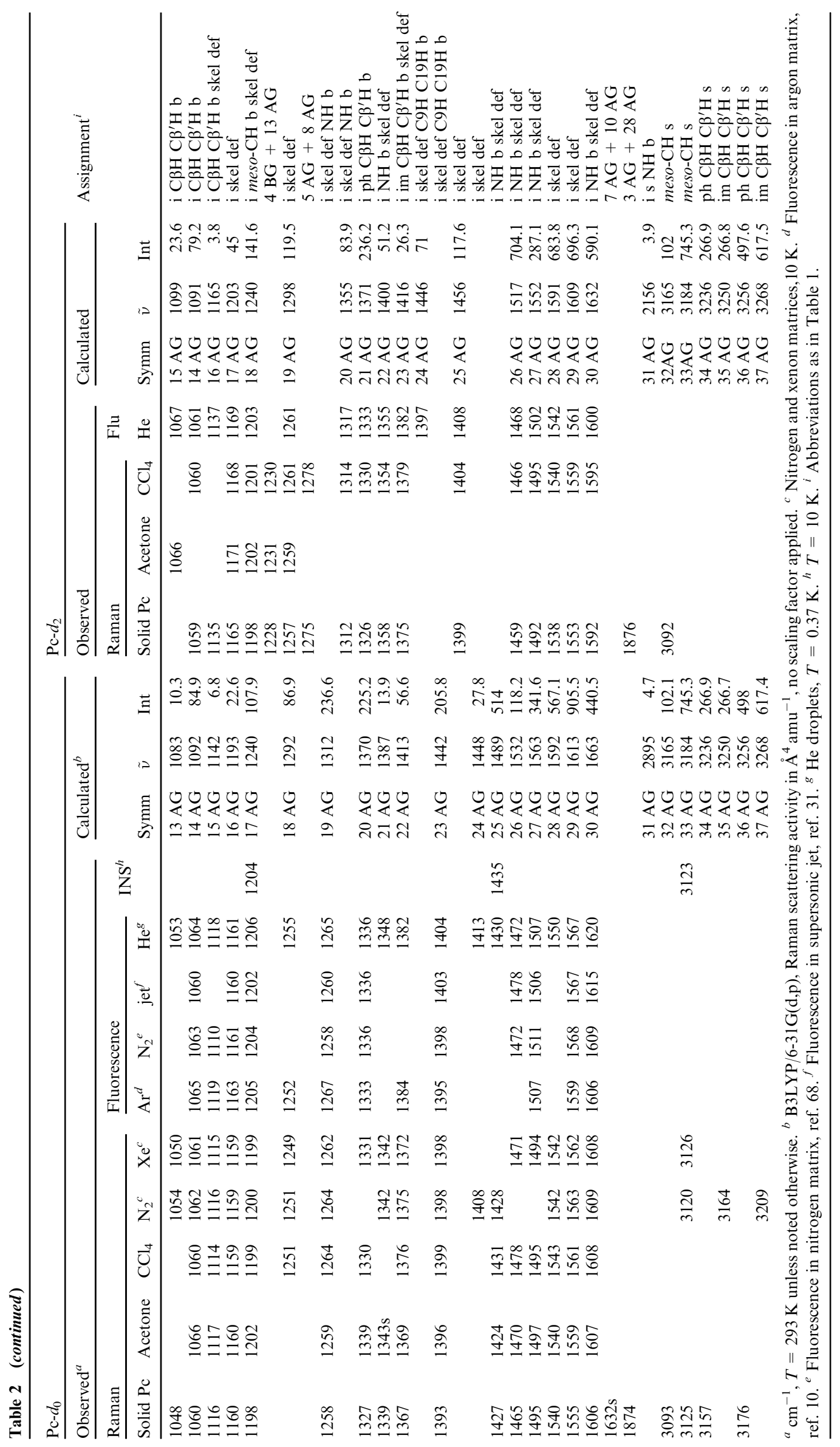




\subsection{Modes of gerade symmetry}

The assignments of vibrations belonging to gerade symmetry species seem even more reliable than those for IR-active modes, because the available experimental database is much larger. The AG and BG vibrations are observed not only in the Raman spectra, but also in fluorescence. Moreover, using different wavelengths for Raman excitation, we were able to switch from a non-resonant to a resonant regime, the latter probing different electronic states. In addition, analysis of depolarization enabled distinguishing between $\mathrm{AG}$ and $\mathrm{BG}$ modes. Finally, some experiments, to be discussed elsewhere in detail, involved SERS or SERRS measurements. Therefore, multiple cross-checks were possible, yielding essentially the same transition energies, but the intensity of different bands responding differently to changes under the conditions of Raman measurements. Finally, as in the case of IR-active transitions, additional support was provided by the INS spectra.

The fluorescence spectrum of Pc- $d_{0}$ embedded in liquid $\mathrm{He}$ droplets (Fig. 3) consists of doublets, which are due to tunneling splitting caused by the quantum delocalization of the internal hydrogens. The splittings are no longer resolved for Pc- $d_{2}$. The spectra are dominated by totally symmetric modes. Thirty AG modes are predicted in the range below $1700 \mathrm{~cm}^{-1}$. All but one could be assigned in the fluorescence spectra for both Pc- $d_{0}$ and Pc- $d_{2}$. The only missing mode, 8 AG, could, however, be readily identified in the Raman spectra, due to its characteristic red shift of $13 \mathrm{~cm}^{-1}$ upon deuteration.

Inspection of Fig. 3 shows that both positions and relative intensities of vibronic components, including overtones and combinations, are nicely predicted by calculations. Only for one mode, $14 \mathrm{AG}$, the computed intensity is considerably lower than the observed ones in both parent and deuterated compounds, even though its location is predicted accurately.

The Raman spectra are shown in Fig. 4 and 5. Most importantly, they not only confirm the assignments of the AG modes, but also provide the positions of the BG modes, which were practically absent in fluorescence. Comparison of spectra obtained with different laser lines, corresponding to both nonresonant $(785 \mathrm{~nm}$ ) and resonant regimes (325 and $514 \mathrm{~nm}$ ), reveals some, but not dramatic, changes in the relative intensities. Some variations in relative intensities are also observed for different environments. In general, the largest intensities in the Raman spectra are observed for totally symmetric AG modes. Analogous situation was reported for Raman spectra of porphyrin obtained for nonresonant excitation; it was explained as indication of the dominance of Franck-Condon over Herzberg-Teller mechanism. ${ }^{58}$

It should be noted that the Raman activities of vibrational modes computed by such software packages as Gaussian are not directly related to the experimental spectrum. A proper comparison requires taking into account the laser frequency and that of a particular vibration, as well as the Boltzmann factor, responsible for the temperature dependence. Such a procedure was applied in this work. As shown in Fig. 4 and 5, the simulated Raman spectra reproduce the experimental pattern quite accurately for both Pc- $d_{0}$ and Pc- $d_{2}$.

On the basis of combined Raman, fluorescence, INS measurements, analysis of isotopic shifts and theoretical studies, we propose the assignments for thirty six out of thirty seven AG modes and for all BG species (Table 2). As was the case for the IR-active modes, we could not observe the vibration corresponding to the $\mathrm{N}-\mathrm{H}$ stretch, $31 \mathrm{AG}$. The $3 \mathrm{BG}$ mode was only identified as an overtone and in combination with $1 \mathrm{AG}$.

Correlation between the experimental and calculated transitions yielded the RMS errors of $7 \mathrm{~cm}^{-1}$ for Pc- $d_{0}$ and $10 \mathrm{~cm}^{-1}$ for Pc- $d_{2}$. As was the case for ungerade species, very similar results were obtained using the $6-31 \mathrm{G}(\mathrm{d}, \mathrm{p})$ and $6-311++\mathrm{G}(\mathrm{fd}, 2 \mathrm{dp})$ basis sets. Again, somewhat larger scaling factors were obtained for the latter, 0.972 for Pc- $d_{0}$ and 0.973 for Pc- $d_{2}$, compared with 0.966 obtained for both isotopologues while using 6-31G(d,p).

Regarding vibrations related to the intramolecular hydrogen bonds, the theory predicts very small intensities for both $\mathrm{N}-\mathrm{H}$ stretching and out-of-plane bending bands. However, we were able to identify the out-of-plane bending mode, 16 BG, which shifts to the red after deuteration by approximately $280 \mathrm{~cm}^{-1}$, the same amount as the corresponding IR-active 18 AU counterpart. Interestingly, the spectral pattern observed after deuteration in the $1100-1600 \mathrm{~cm}^{-1}$ region is not so strongly modified as was the case for the IR spectra.

\subsection{Theoretical modeling and interpretation of the IR spectra}

The patterns of IR spectra of Pc- $d_{0}$ and Pc- $d_{2}$, computed from $a b$ initio molecular dynamics (AIMD) trajectories generated at the BLYP/PW level, compare well with the experimental data in the near infrared region ( $c f$. the top and bottom panels in Fig. 1 and 2). The band locations below $1600 \mathrm{~cm}^{-1}$ are predicted with similar accuracy to those obtained from the static normal mode analysis (NMA) using B3LYP/6-31G(d,p) within harmonic approximation, and the intensity patterns are even better. This reassuring observation provides a premise for applying the AIMD method as a tool to explore the discrepancy between experimental and theoretical results for the $\mathrm{N}-\mathrm{H}$ stretching vibration, where static calculations obviously break down (see Section 3.1). In the following section we confront anharmonic frequencies $\nu_{i}$ of the IR bands computed from the AIMD trajectories (see Section 2 for the definition of the anharmonic frequencies $\nu_{i}$ ) with the experimentally measured values and analyze the underlying molecular motions in order to explain the intriguing features of the IR spectra of porphycene in the high frequency region.

The correction factors, calculated independently for the two isotopologues, Pc- $d_{0}$ and Pc- $d_{2}$, yield $c_{d_{0}}=1.0318$ and $c_{d_{2}}=$ 1.0320 , respectively. Since the experimental data for singly deuterated species, Pc- $d_{1}$, are not available and the difference between $c_{d_{0}}$ and $c_{d_{2}}$ is rather small, we applied one common correction factor $c=1.032$ to all three sets of frequencies, including the one obtained for Pc- $d_{1}$. The correction magnitude for the dynamical BLYP/PW calculations and for the harmonic $\mathrm{B} 3 \mathrm{LYP} / 6-31 \mathrm{G}(\mathrm{d}, \mathrm{p})$ ones is similar. However, the anharmonic BLYP model appears to provide a "softer" potential than that of the actual molecule $(c>1)$, while the potential in the harmonic B3LYP model seems to be too stiff $(c<1)$. Similar difference between two DFT models has been observed before. ${ }^{67}$ The anharmonic frequencies match the experimental ones with the $15 \mathrm{~cm}^{-1}$ standard deviation (an average over Pc- $d_{0}$ and Pc- $d_{2}$ data sets). We conclude that the theoretical 

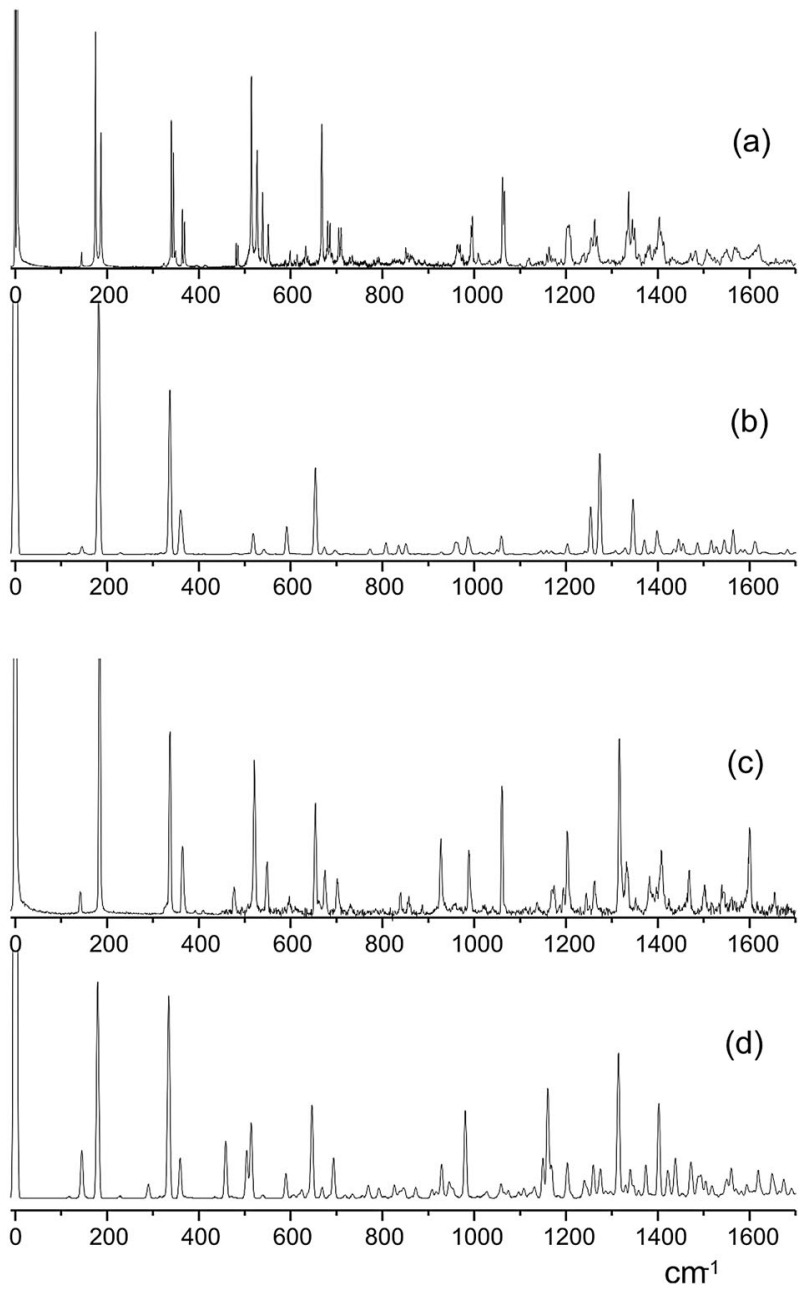

Fig. 3 Dispersed fluorescence of Pc- $d_{0}$ (a) and Pc- $d_{2}$ (c) in helium droplets along with the simulated spectra (b and d).

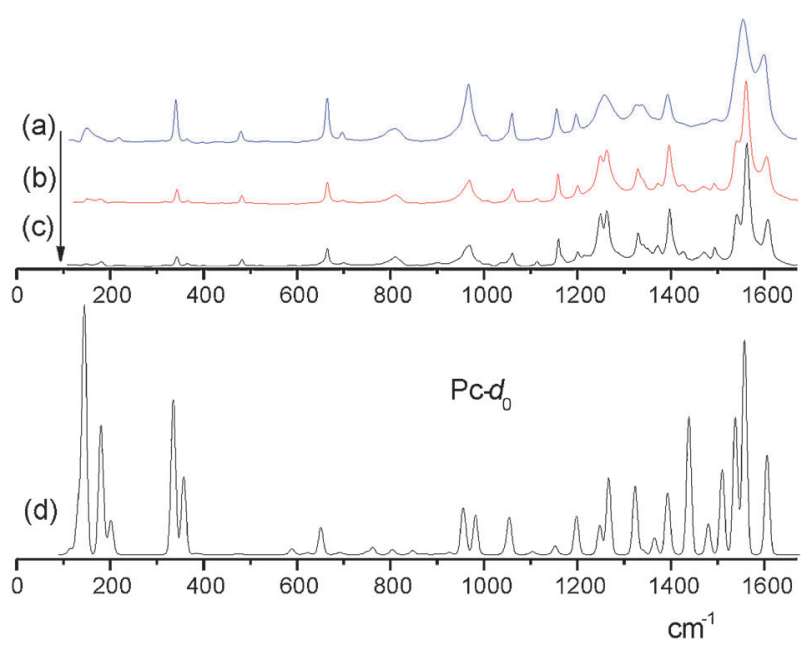

Fig. 4 Top, experimental Raman spectra of Pc- $d_{0}$ obtained with $514 \mathrm{~nm}$ laser line: (a) polycrystalline Pc, $293 \mathrm{~K}$; (b) nitrogen matrix, $10 \mathrm{~K}$; (c) xenon matrix, $10 \mathrm{~K}$; (d) the spectra simulated carrying out B3LYP/ 6-31G $(\mathrm{d}, \mathrm{p})$ calculations, corrected for the laser wavelength and for the temperature of $290 \mathrm{~K}$. Gaussian peaks of $6 \mathrm{~cm}^{-1}$ halfwidths were assumed for all transitions. The scaling factor of 0.966 was used.

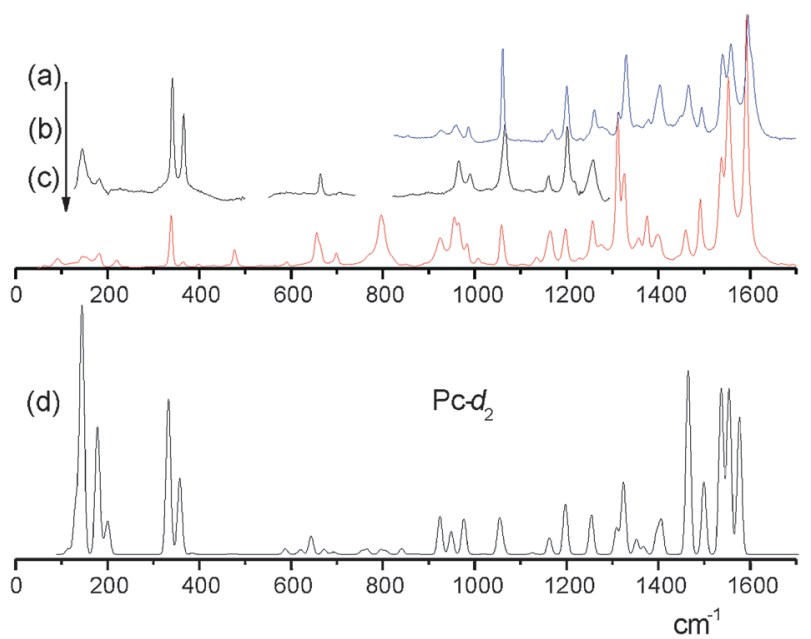

Fig. 5 Top, experimental Raman spectra of Pc- $d_{2}$ at $293 \mathrm{~K}$ obtained with 514 (a and c) or $785 \mathrm{~nm}$ (b) laser lines: (a) $\mathrm{CCl}_{4}$; (b) acetone; (c) polycrystalline sample; (d) the spectra simulated carrying out B3LYP/6-31G(d,p) calculations, corrected for $785 \mathrm{~nm}$ laser wavelength and for the temperature of $290 \mathrm{~K}$. Gaussian peaks of $6 \mathrm{~cm}^{-1}$ halfwidths were assumed for all transitions. The scaling factor of 0.966 was used.

predictions of the IR spectra based on dynamical ab initio calculations are accurate and agree with the experimental measurements within an acceptable error limit.

Crucial for the purpose of the present work are the results obtained for the high-energy range of the IR spectrum (see the bottom panel and horizontal axis in Fig. 6). For the sake of simplicity, in the following discussion we will omit the scaling factors and will refer to the raw frequency values, both harmonic and anharmonic, as obtained from the BLYP/PW computations. Two distinguished features centered at 3100 and $3170 \mathrm{~cm}^{-1}$ result

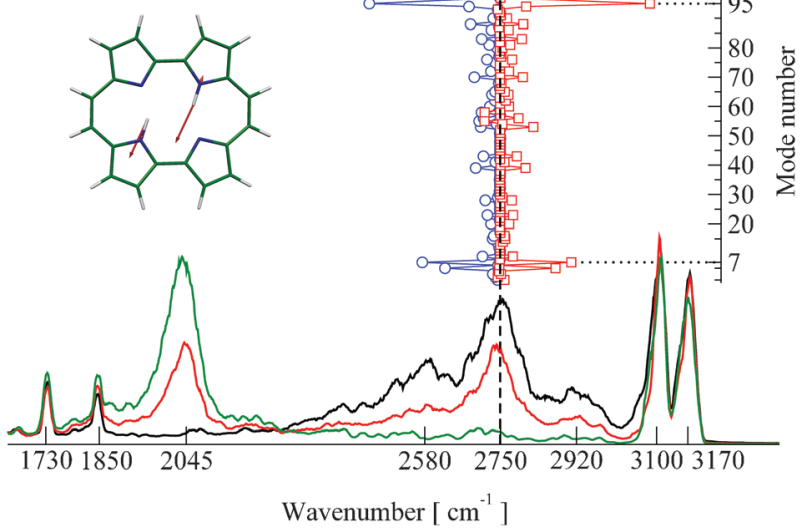

Fig. 6 Computed high frequency range of the IR spectrum of Pc- $d_{0}$ (black line), Pc- $d_{1}$ (red line) and Pc- $d_{2}$ (green line). Harmonic frequency $f_{96}=2753 \mathrm{~cm}^{-1}$ of the asymmetric N-H stretching mode $30 \mathrm{BU}$ (vertical dashed line), perturbed frequencies $f_{96, j}{ }^{-}$(blue circles) and $f_{96, j}{ }^{+}$(red squares) of the $30 \mathrm{BU}$ mode as defined in the text, as a function of a perturbing mode number $j$ (vertical right panel); displacement vector of the asymmetric $\mathrm{N}-\mathrm{H}$ stretching mode $30 \mathrm{BU}$ subject to perturbations from other vibrational modes (left panel). All calculations were carried out using the BLYP/PW exchange-correlation functional, no scaling factor for the frequencies was applied. 


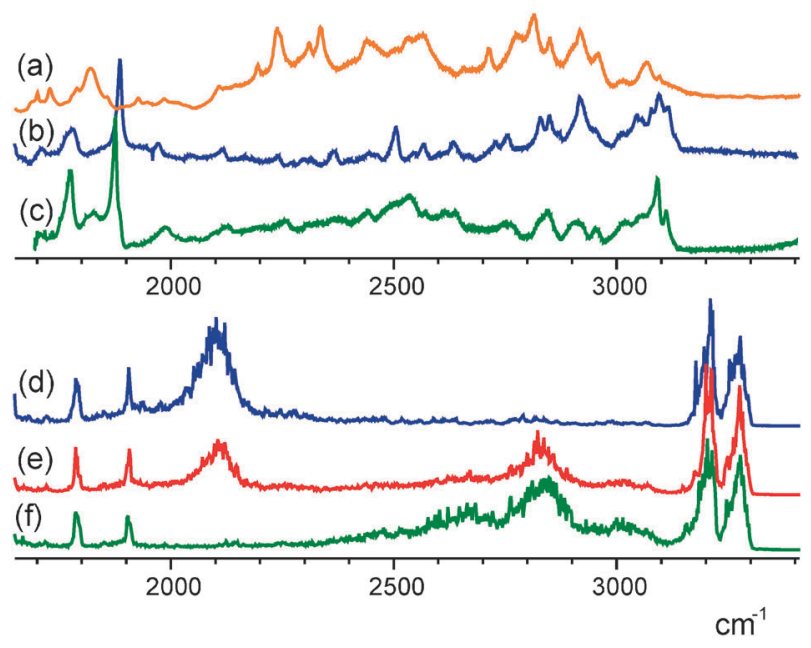

Fig. 7 Top, the experimental IR spectrum recorded at $293 \mathrm{~K}$ for Pc- $d_{12}$ (a), Pc- $d_{2}$ (b) and Pc- $d_{0}$ (c) in KBr. Bottom, IR spectrum of Pc- $d_{2}$ (d), Pc- $d_{1}$ (e) and Pc- $d_{0}$ (f) in the high frequency range as calculated from $a b$ initio molecular dynamics simulations using the BLYP/PW exchange-correlation functional.

from the $\mathrm{C}-\mathrm{H}$ stretching vibrations, with the lower frequency corresponding to the meso $\mathrm{C}-\mathrm{H}$ bonds. As expected, both spectral features due to $\mathrm{C}-\mathrm{H}$ stretches remain unaffected upon deuteration of the two internal hydrogens. This is not only the argument for the correct assignment of the modes, but it also provides an evidence for the motions of the inner hydrogens being decoupled from those of the peripheral ones.

Another two theoretical peaks are located around 1730 and $1850 \mathrm{~cm}^{-1}$. Their positions are not shifted upon deuteration of the internal hydrogen sites. Upon closer inspection each of the two features appears to be composed of two close-lying signals separated by approximately $7 \mathrm{~cm}^{-1}$. The lack of respective counterparts in the harmonic calculations (both B3LYP/ 6-31G(d,p) and BLYP/PW) indicates that these signals are due to combinations or overtones of fundamental vibrational modes. Indeed, excellent agreement is observed between the theoretical and experimental bands for both, Pc- $d_{0}$ and Pc- $d_{2}$ (see Fig. 7).

The positions and intensities of the bands in the infrared region of the spectrum that extends roughly between 1900 and $3000 \mathrm{~cm}^{-1}$ depend on the mass of the inner hydrogen atoms. In the undeuterated species, Pc- $d_{0}$, a broad feature extends over the range of about $750 \mathrm{~cm}^{-1}$ and consists of one main peak centered around $2750 \mathrm{~cm}^{-1}$ and two shoulders-both separated by about $170 \mathrm{~cm}^{-1}$ from the main peak. In singly deuterated species, Pc- $d_{1}$, the integral intensity of this feature is two times smaller than in Pc- $d_{0}$ and a new feature appears around $2045 \mathrm{~cm}^{-1}$, red-shifted by about $700 \mathrm{~cm}^{-1}$ from the main frequency of the first peak. In Pc- $d_{2}$, the high-energy peak fully disappears, while the feature at $2045 \mathrm{~cm}^{-1}$ becomes roughly two times more intense than in Pc- $d_{1}$. This characteristic isotope effect provides evidence that the bands observed in this spectral region result from the motions of the two hydrogen atoms involved in the internal $\mathrm{N}-\mathrm{H} \cdots \mathrm{N}$ hydrogen bonds.

As already mentioned, porphycene has 108 internal degrees of freedom (DOF). In the vicinity of the potential energy minimum they can be approximated by the eigenvectors of the
Hessian matrix, representing normal vibrational modes (NM). In porphycene this harmonic model describes the majority of DOFs quite well. This is reflected by the fact that most of the projected normal mode (PNM) power spectra, $p_{i}(\omega)$, contain single, highly intense peaks, lying close to the corresponding normal mode eigenvalues, $f_{i}$. Due to anharmonicity of the realistic DFT-based potential used for generating the molecular dynamics trajectories, the positions of the peaks are shifted with respect to their harmonic counterparts. The shift amounts to $\sim 10 \mathrm{~cm}^{-1}$ on average, usually towards lower frequencies (red-shift). Only in two cases, i.e. for modes $\boldsymbol{e}_{5}$ and $\boldsymbol{e}_{13}$ assigned to $1 \mathrm{AG}$ and $2 \mathrm{BU}$ vibrations, respectively, a blue shift of a few wavenumbers was observed (see ESI $\dagger$ for the corresponding displacement vectors). In some cases the power spectra contain additional, less intense peaks, mainly in the low frequency region. The additional frequencies in the PNM spectra are either due to numerical noise, e.g., normal mode vectors being not perfectly orthogonal or the inter-mode interactions (see Section 3.4 for details).

There are only two exceptions from the single-peaked shape of the PNM spectra of the undeuterated Pc- $d_{0}$ species: these are modes $\boldsymbol{e}_{95}$ and $\boldsymbol{e}_{96}$ which show extremely broad features in the high frequency region that closely resemble the alreadymentioned band at $2750 \mathrm{~cm}^{-1}$ in the IR spectrum. The underlying molecular motions of the two modes consist of the $\mathrm{N}-\mathrm{H}$ bond stretching vibrations. The lower $\boldsymbol{e}_{95}$ frequency mode corresponds to simultaneous elongation and shortening of the two bonds (in phase, also termed symmetric stretch), while in the higher $\boldsymbol{e}_{96}$ frequency mode one bond gets shortened while the other gets elongated at the same time (in counter-phase, asymmetric stretch). The two BLYP/PW modes correspond to $31 \mathrm{AG}$ and $30 \mathrm{BU}$ vibrations calculated using the harmonic B3LYP/6-31G(d,p) model, with the latter representing the IR active vibration which, unexpectedly, has not been identified in the experimental spectrum (we should note that the excitation of the symmetric $31 \mathrm{AG}$ mode is likely to enhance the concerted proton transfer reaction mechanism, while excitation of the asymmetric $30 \mathrm{BU}$ vibration is expected to drive the stepwise reaction). To shortly summarize the results: so far, PNM-based analysis allowed us to assign the relevant features in the IR spectrum to the specific atomic motions, however, it did not provide insight into their interactions with the other modes that would explain the extreme broadening of the $\mathrm{N}-\mathrm{H}$ stretching band. Inter-mode couplings are discussed in the following paragraph.

\subsection{Inter-mode couplings and broadening of the $\mathrm{N}-\mathrm{H}$ stretching vibration}

In realistic molecular systems the internal degrees of freedom are, in general, interdependent. Deformation of the molecular structure along one DOF is likely to modify the potential energy function in all remaining internal degrees of freedom. Such perturbation would give rise to the force acting on the system along the unperturbed degrees of freedom. Analysis based on this observation has been performed for porphycene using a limited number of modes. ${ }^{35,36}$ Consequently the perturbation of the PES along one normal mode vector would affect the force constants, and thus vibrational frequencies 
associated with the remaining normal modes. In the following we apply the quantitative approach that takes into account couplings of all normal modes to the asymmetric $\mathrm{N}-\mathrm{H}$ stretching mode $30 \mathrm{BU}$ in porphycene (see inset in Fig. 6 for the corresponding displacement vector), however, it can be easily generalized (see ESI $\dagger$ for the details of this method). In a nutshell, the harmonic frequency of the $i$ th normal mode is perturbed by the displacement along another normal mode $j$. The perturbation is quantified by two limiting values $f_{i j}{ }^{+}$and $f_{i j}{ }^{-}$resulting from the displacement in the positive and negative directions along the modulating mode $j$. The magnitude of the perturbation reflects the average amplitude of fluctuations along $j$ at a given temperature.

Normal modes of vibration can be categorized into fast- and slow-varying degrees of freedom, based on their corresponding frequencies. The slow modes are characterized by relatively small curvature of the PES as compared to the fast ones, though the distinction between the two is somewhat arbitrary at this point. As an example, one may consider the slowest normal mode of Pc- $d_{0}-\boldsymbol{e}_{1}$ with eigenfrequency $f_{1}=86 \mathrm{~cm}^{-1}$ which has a period of oscillations $T \approx 400 \mathrm{fs}$, while the fastest one, $\boldsymbol{e}_{108}\left(f_{108}=3198 \mathrm{~cm}^{-1}\right)$ varies on the time scale of $\sim 10 \mathrm{fs}$. Within one vibrational period along the slow DOF the system performs several tens of oscillations along the fast one. As already mentioned, the shape of the PES along the fast mode may depend on the instantaneous displacement along the slow mode. For such a case, the motion along the $j$, the slow DOF modulates the frequency of oscillations along the fast mode $i$, which then becomes a function of the displacement $f_{i}\left(d_{j}\right)$.

Such frequency modulation is indeed observed in porphycene at $300 \mathrm{~K}$. The harmonic frequency of the IR active $\mathrm{N}-\mathrm{H}$ stretching mode $30 \mathrm{BU}$ is significantly perturbed by the coupling to other modes (see vertical panel in Fig. 6 for perturbed frequencies $f_{96, j}{ }^{+}$and $f_{96, j}{ }^{-}$of the mode $30 \mathrm{BU}$ in Pc- $d_{0}$ species as a function of the perturbing mode number $j$ ). The largest perturbation results from the fluctuations along $\boldsymbol{e}_{95}$ $(j=95)$ - the infrared inactive mode $31 \mathrm{AG}$ (see Fig. 8 for the displacement vectors of all relevant normal modes discussed in this section) which modulates the value of $f_{96}$ in the range between 2455 and $3085 \mathrm{~cm}^{-1}$, spanning $630 \mathrm{~cm}^{-1}$ and being sensitive to the inner H/D substitution. It should be pointed out that the normal frequencies of both, the perturbed mode $30 \mathrm{BU}$ and the perturbing mode $31 \mathrm{AG}$, are only few wavenumbers apart and therefore they cannot be considered fast and slow with respect to each other. It implies that the simple concept of frequency modulation may not be fully appropriate to explain the mechanism of the inter-mode interaction in this case. However, the existence of such interaction is undeniable.

There are two other modes that introduce significant perturbation to $f_{96}$. The normal mode $2 \mathrm{AG}(j=7)$, widely discussed $^{18,27,35,36}$ in the context of the mechanism of the double proton transfer reaction in porphycene, modulates the fundamental frequency of the $30 \mathrm{BU}$ mode within the range of $334 \mathrm{~cm}^{-1}$, between $2575 \mathrm{~cm}^{-1}$ and $2910 \mathrm{~cm}^{-1}$. Interestingly the shoulders associated with the main $\mathrm{N}-\mathrm{H}$ signal discussed in Section 3.3 lie precisely on the boundaries of this range ( $c f$. black curve in the horizontal panel and open symbols in the vertical panel of Fig. 6). Perturbation of $f_{96}$ due to the mode $1 \mathrm{AG}(j=5)$ is enclosed within 2625 and

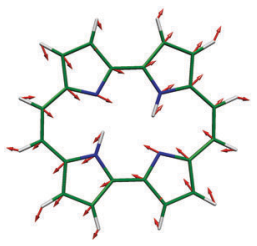

$1 \mathrm{AG}, 150 \mathrm{~cm}^{-1}$

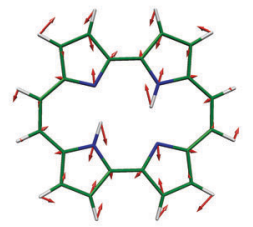

$11 \mathrm{AG}, 994 \mathrm{~cm}^{-1}$

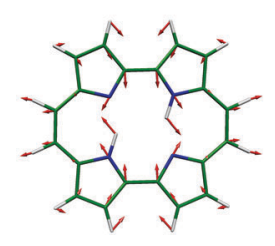

$2 \mathrm{AG}, 187 \mathrm{~cm}^{-1}$

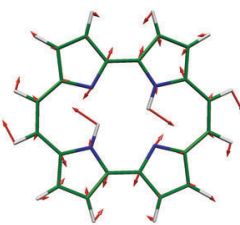

$19 \mathrm{AG}, 1312 \mathrm{~cm}^{-1}$

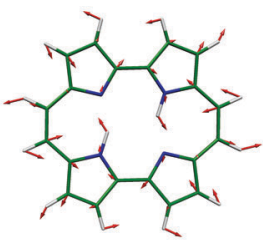

$8 \mathrm{AG}, 833 \mathrm{~cm}^{-1}$

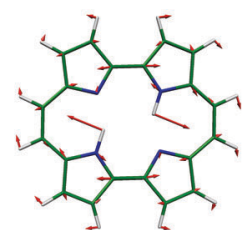

$30 \mathrm{AG}, 1663 \mathrm{~cm}^{-1}$

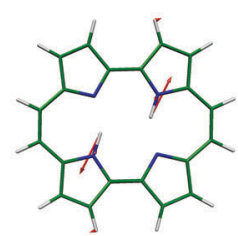

$31 \mathrm{AG}, 2895 \mathrm{~cm}^{-1}$

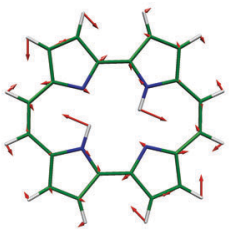

$27 \mathrm{AG}, 1563 \mathrm{~cm}^{-1}$

Fig. 8 Displacement vectors of the normal modes in Pc- $d_{0}$ relevant to the broadening of the asymmetric $\mathrm{N}-\mathrm{H}$ stretching mode $30 \mathrm{BU}$, as obtained with B3LYP/6-31G(d,p).

$2873 \mathrm{~cm}^{-1}$-spanning the range of $248 \mathrm{~cm}^{-1}$. Other five normal modes, 8 AG $(j=39), 11$ AG $(j=53), 19$ AG $(j=70), 27 \mathrm{AG}(j=88)$, and $30 \mathrm{AG}(j=94)$, contribute more than $100 \mathrm{~cm}^{-1}$ to the broadening of the $f_{96}$ peak. The majority of all modes, i.e. 74 out of 108 , do not couple to the mode $30 \mathrm{BU}$ since the broadening of the fundamental frequency $f_{96}$ due to the activation of those modes is smaller than $10 \mathrm{~cm}^{-1}$. In conclusion, the low, if not undetectable, intensity of the $\mathrm{N}-\mathrm{H}$ stretching vibration in the experimental IR spectra results from the broadening caused by other modes. Coupling of the asymmetric $30 \mathrm{BU}$ mode to the low frequency $7 \mathrm{AG}$ mode was discussed previously by many authors ${ }^{18,27,35,36}$ and our results confirm nicely their findings. The extended analysis presented here reveals other additional modes that contribute significantly to the broadening of the IR $\mathrm{N}-\mathrm{H}$ stretching vibration (Fig. 8).

We close the discussion on inter-mode interactions with the remark that the results presented here were obtained from the full-dimensional PES obtained at the ab initio level and without making any assumption on its shape. The harmonic ansatz was invoked only to decompose the complex molecular motions into easy-to-visualize components, and to quantify the coupling effects.

\section{Summary and conclusions}

Using combined experimental and theoretical procedures resulted in the assignments of 105 out of 108 vibrations of porphycene. DFT seems, especially when combined with isotopic substitution, to be a good method for a reliable analysis of vibrational structure. However, it is clear that the analysis of strong hydrogen bonds requires a special treatment. 
The results of theoretical analysis, which goes beyond the harmonic approximation, allowed the interpretation of the IR spectra. We postulate that the apparent lack of the $\mathrm{N}-\mathrm{H}$ stretching band in the experimental spectra, predicted by harmonic calculations to be the strongest in the IR spectrum, is caused by its extreme breadth. The integrated intensity is very high, but the height at maximum is not. The large spectral width is due to the interaction of the $\mathrm{N}-\mathrm{H}$ stretch with other modes, in particular those which alter the $\mathrm{N} \cdots \mathrm{N}$ distance, and thus the HB strength. One can envisage the frequency of the $\mathrm{N}-\mathrm{H}$ stretch as being continuously modulated by the low frequency modes.

The unequivocal experimental confirmation of this model may not be easy. The experimental IR spectra (Fig. 7) clearly show an extremely broad band in the range of $2000-3000 \mathrm{~cm}^{-1}$, with many structured features superimposed upon the underlying continuum. The maximum of the broad band lies around $2500 \mathrm{~cm}^{-1}$, about $200 \mathrm{~cm}^{-1}$ lower than predicted by theory. An analogous broad feature has been observed for Pc- $d_{12}$, but not for Pc- $d_{2}$. Interestingly, for the latter theory predicts a much narrower band.

Our results may be relevant for the elucidation of the mechanism of double hydrogen transfer in porphycenes. The experiments performed for porphycenes isolated in the jet or embedded in helium droplets revealed tunneling splittings of which the values were strongly mode-dependent. ${ }^{15,20,31}$ The present findings clearly indicate the multidimensional character of the internal hydrogens motions. Time-resolved vibrational spectroscopy experiments in the condensed phase are planned, with the goal of obtaining a detailed picture of intramolecular mode coupling.

\section{Acknowledgements}

We appreciate the help of Samantha Ford in neutron scattering experiments. $\mathrm{EW}$ is grateful to Harald Forbert for valuable discussions. The work was supported by the European Union within European Regional Development Fund, through the Innovative Economy grant (POIG.01.01.02-00-008/08) and by the Polish Ministry of Science and Higher Education (N N519 384736). The simulations were carried out at ICM University of Warsaw (Halo2), and in Forschungszentrum Jülich (Juropa). The INS experiments were supported by the Institut LaueLangevin proposal 7-04-83.

\section{Notes and references}

1 Handbook of Porphyrin Science, ed. K. Smith, K. Kadish and R. Guilard, World Scientific, Singapore, 2010.

2 P. F. Aramendia, R. W. Redmond, S. Nonell, W. Schuster, S. E. Braslavsky, K. Schaffner and E. Vogel, Photochem. Photobiol., 1986, 44, 555-559.

3 J. Waluk, M. Müller, P. Swiderek, M. Köcher, E. Vogel, G. Hohlneicher and J. Michl, J. Am. Chem. Soc., 1991, 113, $5511-5527$.

4 J. C. Stockert, M. Cañete, A. Juarranz, A. Villanueva, R. W. Horobin, J. Borrell, J. Teixidó and S. Nonell, Curr. Med. Chem., 2007, 14, 997-1026.

5 J. Braun, H. H. Limbach, P. G. Williams, H. Morimoto and D. E. Wemmer, J. Am. Chem. Soc., 1996, 118, 7231-7232.

6 J. Braun, M. Schlabach, B. Wehrle, M. Köcher, E. Vogel and H. H. Limbach, J. Am. Chem. Soc., 1994, 116, 6593-6604.

7 M. Schlabach, B. Wehrle, H. Rumpel, J. Braun, G. Scherer and H. H. Limbach, Ber. Bunsen-Ges. Phys. Chem., 1992, 96, 821-833.
8 B. Wehrle, H. H. Limbach, M. Köcher, O. Ermer and E. Vogel, Angew. Chem., Int. Ed. Engl., 1987, 26, 934-936.

9 P. M. Kozlowski, M. Z. Zgierski and J. Baker, J. Chem. Phys., 1998, 109, 5905-5913.

10 K. Malsch and G. Hohlneicher, J. Phys. Chem. A, 1997, 101, 8409-8416.

11 J. Baker, P. M. Kozlowski, A. A. Jarzecki and P. Pulay, Theor. Chem. Acc., 1997, 97, 59-66.

12 H. Piwoński, C. Stupperich, A. Hartschuh, J. Sepioł, A. Meixner and J. Waluk, J. Am. Chem. Soc., 2005, 127, 5302-5303.

13 U. Langer, C. Hoelger, B. Wehrle, L. Latanowicz, E. Vogel and H. H. Limbach, J. Phys. Org. Chem., 2000, 13, 23-34.

14 M. Gil, J. Jasny, E. Vogel and J. Waluk, Chem. Phys. Lett., 2000, 323, 534-541.

15 J. Sepioł, Y. Stepanenko, A. Vdovin, A. Mordziński, E. Vogel and J. Waluk, Chem. Phys. Lett., 1998, 296, 549-556.

16 T. Yoshikawa, S. Sugawara, T. Takayanagi, M. Shiga and M. Tachikawa, Chem. Phys. Lett., 2010, 496, 14-19.

17 J. Waluk, in Handbook of Porphyrin Science, ed. K. Smith, K. Kadish and R. Guilard, World Scientific, Singapore, 2010, vol. 7, pp. 359-436.

18 Ł. Walewski, J. Waluk and B. Lesyng, J. Phys. Chem. A, 2010, 114, 2313-2318.

19 M. Gil, J. Dobkowski, G. Wiosna-Sałyga, N. Urbańska, P. Fita, C. Radzewicz, M. Pietraszkiewicz, P. Borowicz, D. Marks, M. Glasbeek and J. Waluk, J. Am. Chem. Soc., 2010, 132, 13472-13485.

20 A. Vdovin, J. Waluk, B. Dick and A. Slenczka, ChemPhysChem, 2009, 10, 761-765.

21 J. Lopez del Amo, U. Langer, V. Torres, M. Pietrzak, G. Buntkowsky, H. M. Vieth, M. F. Shibl, O. Kühn, M. Bröring and H. H. Limbach, J. Phys. Chem. A, 2009, 113, 2193-2206.

22 P. Fita, N. Urbańska, C. Radzewicz and J. Waluk, Chem.-Eur. J., $2009,15,4851-4856$.

23 J. Waluk, Pol. J. Chem., 2008, 82, 947-962.

24 Z. Smedarchina, W. Siebrand, A. Fernandez-Ramos and R. Meana-Paneda, Z. Phys. Chem. (Muenchen, Ger.), 2008, 222, 1291-1309.

25 P. Fita, N. Urbańska, C. Radzewicz and J. Waluk, Z. Phys. Chem., 2008, 222, 1165-1173.

26 J. Waluk, in Hydrogen-Transfer Reactions, ed. J. T. Hynes, J. P. Klinman, H. H. Limbach and R. L. Schowen, Wiley-VCH, Weinheim, 2007, vol. 1, pp. 245-271.

27 Z. Smedarchina, M. F. Shibl, O. Kühn and A. Fernández-Ramoz, Chem. Phys. Lett., 2007, 426, 314-321.

28 M. Pietrzak, M. F. Shibl, M. Bröring, O. Kühn and H. H Limbach, J. Am. Chem. Soc., 2007, 129, 296-304.

29 M. Gil and J. Waluk, J. Am. Chem. Soc., 2007, 129, 1335-1341.

30 J. Waluk, Acc. Chem. Res., 2006, 39, 945-952.

31 A. Vdovin, J. Sepioł, N. Urbańska, M. Pietraszkiewicz, A. Mordziński and J. Waluk, J. Am. Chem. Soc., 2006, 128, 2577-2586.

32 P. Fita, P. Garbacz, M. Nejbauer, C. Radzewicz and J. Waluk, Chem.-Eur. J., 2011, 17, 3672-3678.

33 H. Piwoński, A. Hartschuh, N. Urbańska, M. Pietraszkiewicz, J. Sepioł, A. Meixner and J. Waluk, J. Phys. Chem. C, 2009, 113 11514-11519.

34 M. K. Abdel-Latif and O. Kühn, Theor. Chem. Acc., 2011, 128 307-316.

35 M. F. Shibl, M. Pietrzak, H. H. Limbach and O. Kühn, ChemPhysChem, 2007, 8, 315-321.

36 M. F. Shibl, M. Tachikawa and O. Kühn, Phys. Chem. Chem. Phys., 2005, 7, 1368-1373.

37 R. M. Gulam, T. Matsushita, S. Neya, N. Funasaki and J. Teraoka, Chem. Phys. Lett., 2002, 357, 126-130.

38 R. M. Gulam, T. Matsushita and J. Teraoka, J. Phys. Chem. A, 2003, 107, 2172-2178.

39 R. M. Gulam, S. Neya and J. Teraoka, J. Porphyrins Phthalocyanines, 2006, 10, 1271-1284.

40 M. G. Rabbani and J. Teraoka, Spectrochim. Acta, Part A, 2010, 76, 207-212.

41 N. Urbańska, M. Pietraszkiewicz and J. Waluk, J. Porphyrins Phthalocyanines, 2007, 11, 596-600.

42 E. Vogel, M. Köcher, H. Schmickler and J. Lex, Angew. Chem., Int. Ed. Engl., 1986, 25, 257-259.

43 J. P. Toennies and A. F. Vilesov, Annu. Rev. Phys. Chem., 1998, 49, $1-41$ 
44 J. P. Toennies and A. F. Vilesov, Angew. Chem., Int. Ed., 2004, 43, $2622-2648$.

45 M. Leverenz, B. Schilling and J. P. Toennies, Chem. Phys. Lett., 1993, 206, 381-387.

46 NIST Atomic Spectra Database (version 3.1.5), http://physics.nist. gov/asd3. National Institute of Standards and Technology, Gaithersburg, MD, USA.

47 http://www.ill.eu/instruments-support/instruments-groups/instruments/ in $4 /$.

$48 \mathrm{http}: /$ www.ill.eu/instruments-support/instruments-groups/instruments/ in $1 /$.

49 http://www.ill.eu/instruments-support/computing-for-science/ cs-software/all-software/lamp/the-lamp-book/.

50 CPMD version 3.13.2, http://www.cpmd.org/, Copyright IBM Corp. 1990-2008, Copyright MPI für Festkörperforschung Stuttgart 1997-2001.

51 A. D. Becke, Phys. Rev. A, 1998, 38, 3098-3100.

52 C. L. Lee, W. Yang and R. G. Parr, Phys. Rev. B: Condens. Matter, 1988, 37, 785-789.

53 N. Troullier and J. L. Martins, Phys. Rev. B: Condens. Matter, 1991, 43, 1993-2006.

54 R. W. Hockney, Methods Comput. Phys., 1970, 9, 135-211.

55 G. J. Martyna, M. L. Klein and M. Tuckerman, J. Chem. Phys., 1992, 97, 2635-2643.

56 R. Ramirez, T. Lopez-Ciudad, P. Kumar and D. Marx, J. Chem. Phys., 2004, 121, 3973-3983.
57 P. M. Kozlowski, A. A. Jarzcki and P. Pulay, J. Phys. Chem., 1996, 100, 7007-7010.

58 P. M. Kozlowski, A. A. Jarzcki, P. Pulay, X.-Y. Li and M. Z. Zgierski, J. Phys. Chem., 1996, 100, 13985-13992.

59 N. Verdal, P. M. Kozlowski and B. S. Hudson, J. Phys. Chem. A, 2005, 109, 5724-5733.

60 D. C. Bieńko, D. Michalska, S. Roszak, W. Wojciechowski, M. J. Nowak and L. Lapinski, J. Phys. Chem. A, 1997, 101, 7834-7841.

61 L. Lapinski, M. J. Nowak, D. C. Bieńko and D. Michalska, Phys. Chem. Chem. Phys., 2002, 4, 1123-1128.

62 T. Stepanenko, L. Lapinski, M. J. Nowak, J. S. Kwiatkowski and J. Leszczynski, Spectrochim. Acta, Part A, 2001, 57, 375-383.

63 S. Gawinkowski, J. Eilmes and J. Waluk, J. Mol. Struct., 2010, 976, 215-225.

64 J. Radziszewski, M. Nepraš, V. Balaji, J. Waluk, E. Vogel and J. Michl, J. Phys. Chem., 1995, 99, 14254-14260.

65 J. G. Radziszewski, J. Waluk and J. Michl, Chem. Phys., 1989, 136, $165-180$

66 S. F. Mason, J. Chem. Soc., 1958, 976-982.

67 M. D. Halls, J. Velkovski and H. B. Schlegel, Theor. Chem. Acc., 2001, 105, 413-421.

68 A. Starukhin, E. Vogel and J. Waluk, J. Phys. Chem. A, 1998, 102, 9999-10006.

69 G. J. Martyna and M. E. Tuckerman, J. Chem. Phys., 1999, 110 $2810-2821$. 